\title{
RECALIBRATION OF A WALL-CURRENT MONITOR USING A FARADAY CUP FOR THE KEKB INJECTOR LINAC
}

\author{
T. Suwada $^{\#}$, S. Ohsawa, K. Furukawa, N. Akasaka, and K. Oide \\ Accelerator Laboratory, High Energy Accelerator Research Organization (KEK) \\ 1-1 Oho, Tsukuba, Ibaraki 305-0801, Japan
}

\section{Abstract}

An absolute beam-charge measurement of single-bunch electron beams with a pulse width of 10 ps and shortpulsed electron beams with a pulse width of 1 ns was performed by the beam test for the KEKB injector linac using a Faraday cup. A wall-current monitor was directly recalibrated by the beam test with an error of $\pm 2 \%$.

\section{INTRODUCTION}

The KEK B-Factory (KEKB) [1] is an asymmetric electron-positron collider comprising $3.5-\mathrm{GeV}$ positron and $8-\mathrm{GeV}$ electron rings. The KEKB injector linac [2] was upgraded in order to inject single-bunch positron and electron beams directly into the KEKB rings. The beam charges are required to be $0.64 \mathrm{nC} /$ bunch and 1.3 $\mathrm{nC} /$ bunch for the positron and electron beams, respectively. High-current primary electron beams $(\sim 10$ $\mathrm{nC} /$ bunch) are required in order to generate sufficient positrons. About seventy wall-current monitors (WCMs) have been newly installed in order to reinforce the beamcharge-monitoring system in the injector linac for the KEKB. A bench calibration for the WCMs was performed using fast test pulses with a width of nanoseconds, and the calibration coefficients were derived from the pulse-height response of the monitor, depending upon the pulse width [3]. It is, however, difficult to directly obtain the calibration coefficient for a single-bunch beam with a pulse width of about $10 \mathrm{ps}$, because the direct generation of such extremely fast test pulses is not very easy. One of the authors (T. Suwada) has tentatively derived calibration coefficients for a 10-ps pulse width from extrapolation based on the results of the bench calibration. It is, however, not sufficiently accurate to estimate them by this extrapolation method, because the WCM has a strong frequency response and a beam-position dependence for shorter pulses [4]. A recalibration of the WCM using a Faraday cup (FC) has been performed using beam tests. The purpose of this report is to give the results of precise measurements of the amount of an absolute beam charge for a single-bunch beam with a pulse width of $10 \mathrm{ps}$ and a short-pulsed beam with a pulse width of $1 \mathrm{~ns}$ generated from the injector linac, and the recalibration for the WCM by a beam test.

\# Email: tsuyoshi.suwada@kek.jp

\section{FARADAY CUP}

A cross-sectional view of the FC is shown in Fig.1.

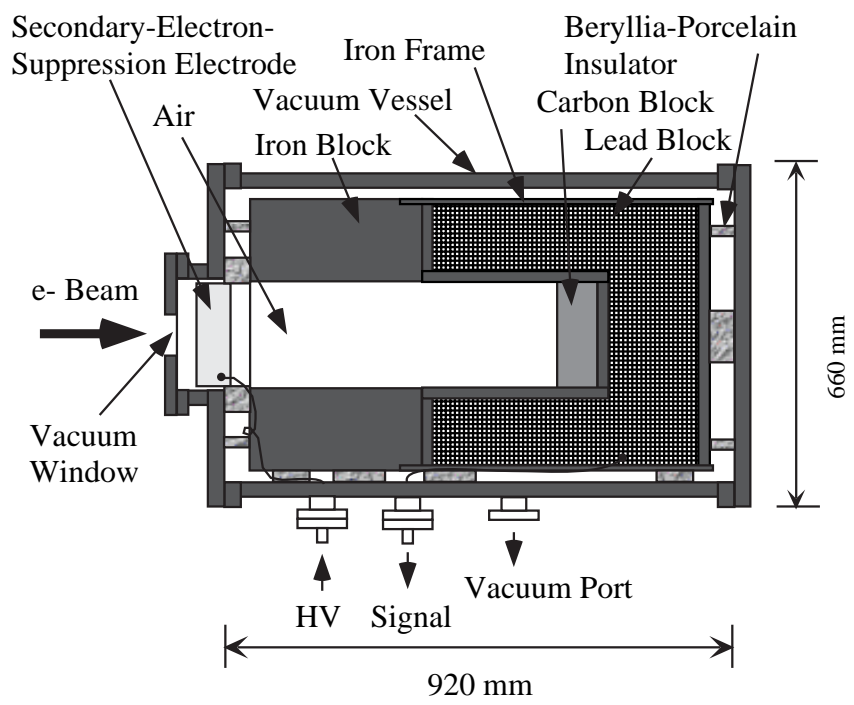

Figure 1: Cross-sectional view of the Faraday cup.

It was originally designed at the Laboratory of Nuclear Science of Tohoku University about thirty years ago. Since a detailed design report is available elsewhere [5], here, the geometry and a simple discussion about its design are briefly presented. The FC comprises a vacuum vessel made of iron, a lead block, an iron block and a carbon block with cylindrical symmetry. The FC dimensions are about $1 \mathrm{~m}$ in total length to the beam axis and about $660 \mathrm{~mm}$ in diameter; the total weight is about $1.8 \mathrm{t}$. The bottom thickness of the lead block is $190 \mathrm{~mm}$, which corresponds to 34 radiation lengths, in order to perfectly absorb incident electron beams with an energy of less than $300 \mathrm{MeV}$. The FC can be separated by the vacuum vessel and a thin vacuum window with a thickness of $0.01 \mathrm{~mm}$ made of stainless steel (SUS304) from the beam line. The window thickness is required to be as thin as possible in order to suppress as much as possible any backscattering and multiplescattering of incident beams. A carbon block with a thickness of 80 $\mathrm{mm}$ is used to suppress any rapid increase of an electromagnetic shower cascade caused by incident beams in the lead block. An opening hole with a diameter of $200 \mathrm{~mm}$ and a depth of $600 \mathrm{~mm}$ in the central region needs to suppress the escape of secondary charged particles 
generated by an electromagnetic shower cascade in the carbon block. A good vacuum condition needs to be maintained through a vacuum port in order to suppress the generation of ions caused in the residual air gas. The inner materials are sufficiently insulated to be larger than 1000 $\mathrm{M} \Omega$ by several beryllia porcelains from the vacuum vessel, which is connected to a ground line. A negative electric potential with several hundred volts is typically applied to an electrode at the entrance of the monitor through a vacuum feedthrough in order to suppress the escape of directly backscattered charged particles as much as possible. The integrated beam charge is extracted from a signal port, which is connected to the lead block.

\section{EXPERIMENTAL SET-UP}

Figure 2 shows a schematic layout of the experimental set-up and the beam line at sectors A and B of the injector linac.

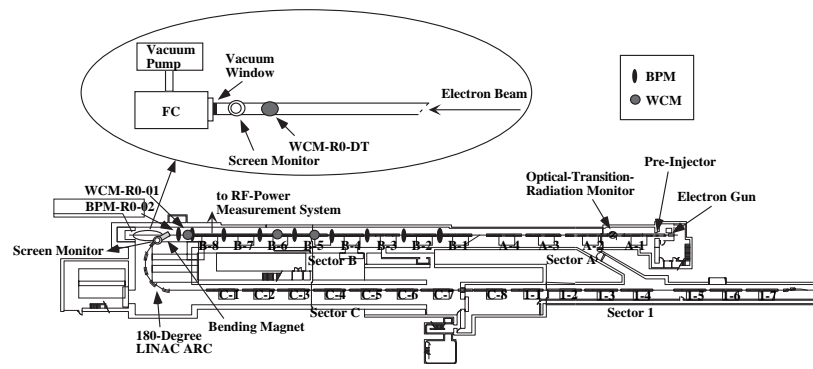

Figure 2: Schematic layout of the experimental set-up and the beam line for the sectors $\mathrm{A}$ and $\mathrm{B}$ at the injector linac.

Single-bunch electron beams can be generated by a new pre-injector [6], which is composed of two sub-harmonic bunchers, a prebuncher and a buncher. The electron gun can generate a beam charge of about $20 \mathrm{nC} /$ pulse. The nominal beam energies are about $500 \mathrm{MeV}$ and $1.5 \mathrm{GeV}$ at the end of the sectors $\mathrm{A}$ and $\mathrm{B}$, respectively. $\mathrm{A}$ longitudinal beam profile can be monitored using an optical transition-radiation monitor (OTR) with a streakcamera system [7] installed after the exit of the module A1. The beam experiments were carried out using electron beams ejected from a straight line after the sector $\mathrm{B}$. The FC was installed $2.3 \mathrm{~m}$ behind the first bending magnet, which guides the electron beams to the 180-degree arc section. The beam energies and the energy spreads were measured after the bending magnet using a screen monitor installed behind. The vacuum of the FC was separated from the beam line with a $10 \mu \mathrm{m}$-thick vacuum window $30 \mathrm{~mm} \phi$ in diameter made of stainless steel, and the vacuum pressure inside the FC was maintained at $1.1 \times 10^{-4}$ Torr using a turbo-molecular vacuum pump. Twenty-two stripline-type beam-position monitors (BPMs) and twenty WCMs measure the beam positions and the beam charge in order to control them stable without any beam loss, respectively. Another WCM and a screen monitor were mounted just before the FC in order to observe the beam losses and the spatial beam sizes, respectively. A data- acquisition (DAQ) system was constructed for the beam experiment. It comprises several front-end computers and a host computer, which control the BPMs and the WCMs, an electrometer (Keithley 617) used for the FC. All of the BPMs and the WCMs are controlled by digital sampling oscilloscopes (Tektronics TDS680B) and VME/OS-9based front-end computers. The host computer and the front-end computers communicate with each other through a network system. Trigger-pluses synchronized with the linac beam are provided to all of the devices at $0.33 \mathrm{~Hz}$. This rate is limited by the communication throughput between the front-end computer and each device through a GPIB line. A detailed report is available elsewhere [8].

\section{BEAM TEST}

\subsection{Beam experiment}

Two kinds of electron beams, single-bunch and shortpulsed beams with the energies of $1.5 \mathrm{GeV}$ and $325 \mathrm{MeV}$, were tuned for the beam test. Single-bunch beams were generated using the two sub-harmonic bunchers; on the other hand, short-pulsed beams were accelerated without them. A single-bunch beam with the energy of $1.5 \mathrm{GeV}$ is a nominal-injection beam for the KEKB ring. The other beams with the energy of $325 \mathrm{MeV}$ were generated in order to reduce as much as possible any systematic error for the beam-charge measurement. The energy spread of the $325-\mathrm{MeV}$ beam was $5.5 \%$, which was greater than that $(2 \%)$ of the $1.5-\mathrm{GeV}$ beam due to the longitudinal beam spread caused by a deacceleration at the sector B. The bunch length of the single-bunch beam and the envelope width of the short-pulsed beam were measured by the OTR to be 8.64 ps and $1 \mathrm{~ns}$ in FWHM, respectively. The beam orbits were carefully tuned for all of the beam modes by using the BPMs without any observational beam loss. The spatial beam size was measured to be about $6 \mathrm{~mm}$ in diameter in front of the FC by a screen monitor. The negative bias voltage applied to the secondary-electronsuppression electrode was chosen to be $-1 \mathrm{kV}$ by measuring the beam-charge reduction using single-bunch beams with the energy of $1.5 \mathrm{GeV}$ by changing the applied voltage. Two background measurements were carried out before the beam-charge measurement. The first was a dark current generated from accelerator structures and the second was a dark current from the electron gun. The first background was estimated by the beam-charge reduction by disturbing the dark currents using a screen monitor in front of the FC with a "beam off" condition, which meant no high voltage applied to the electron gun. The second background was obtained by measuring the beam-charge increment with a "beam wait" condition, which meant no triggers were fed to the gun with a high voltage applied. The background contribution from the first one was negligibly small compared with the second one $(0.3 \mathrm{nC} / \mathrm{scan})$ which was corrected in the analysis. The beam-charge measurement was carried out by 
changing the bias voltage applied to the gun four times, which were 172.8, 191.2, 209.6 and $228.4 \mathrm{~V}$; the measured beam charge was about 1 to $3 \mathrm{nC}$ for all of the beam modes.

\subsection{Experimental results}

It is important to carefully analyze the beam loss between the end of the module B- 8 and the FC, any generation of secondary charged particles (shower) at the vacuum window, and the effect of a beam halo, because such phenomena may produce systematic errors. The first and second phenomena can be estimated by relating the ratios of the charge measured by the FC to that of the WCMR0-01, and to that of the WCM-R0-DT depnding upon the beam positions obtained by the BPM-R0-02 (see Fig.2). The systematic error analysis is given elsewhere in detail [9]. Figure 3 show the results after only a dark-current correction for a $1.5-\mathrm{GeV}$ single-bunch beam. We can find beam losses slightly before the FC in the region of the horizontal beam-position displacement of less than zero. An analysis was performed after data reduction due to the beam loss and after rejecting data larger than $\pm 2 \sigma$ obtained from a Gaussian-function fitting procedure for the chargeratio distributions in order to exclude any noisy events caused by the klystron modulators. Figure 4 shows a linear relation of the beam charge of the WCM-R0-01 to that of the FC by using a least-squares fitting procedure after data reduction. The slope (a) corresponds to a recalibration coefficient of the beam charge for the WCMR0-01. The errors in the figure give only statistical errors. For the other beam modes, the analysis was also performed in the same way after the data-reduction procedure while carefully taking into account the beamloss and the shower events. Table 1 summarizes the analysis results for all of the beam modes and the combined recalibration coefficients for the single-bunch (sb) beam, and for the short-pulsed (s-p) beam, respectively.

\section{CONCLUSIONS}

An absolute beam-charge measurement for single-bunch and short-pulsed electron beams was performed by using a Faraday cup at the KEKB injector linac. A wall-current monitor was recalibrated according to the analysis results; the recalibration coefficients were obtained to be $1.11 \pm$ $7 \times 10^{-4}$ (stat.) $\pm 2 \times 10^{-2}$ (syst.) for the single-bunch beam, and $1.04 \pm 2 \times 10^{-3}$ (stat.) $\pm 2 \times 10^{-2}$ (syst.) for the short-pulsed beam, respectively.

\section{ACKNOWLEDGMENTS}

We are grateful to Prof. T. Terasawa (Tohoku University, Japan) for allowing us the use of his group's Faraday cup.

\section{REFERENCES}

[1] KEKB B-Factory Design Report:KEK Report 95-7 (1995).

[2] Design Report on PF Injector Linac Upgrade for KEKB: KEK Report 95-18, 1996.

[3] T. Suwada et al.,Nucl. Instrum. \& Methods A396 (1997) p.1.

[4] T. Suwada et al., APAC'98, KEK, Tsukuba, Japan, 1998, p.528.

[5] T. Ohama et al., Mitsubishi Electric Advance 43 (5) p.739 (1969).

[6] S. Ohsawa et al., LINAC'96, CERN, Geneva, Switzerland, 1996, p.815.

[7] Y. Ogawa et al., APAC'98, KEK, Tsukuba, Japan, 1998, p.534.

[8] T. Suwada et al., APAC'98, KEK, Tsukuba, Japan, 1998, p.531.

[9] T. Suwada, to be in preparation.

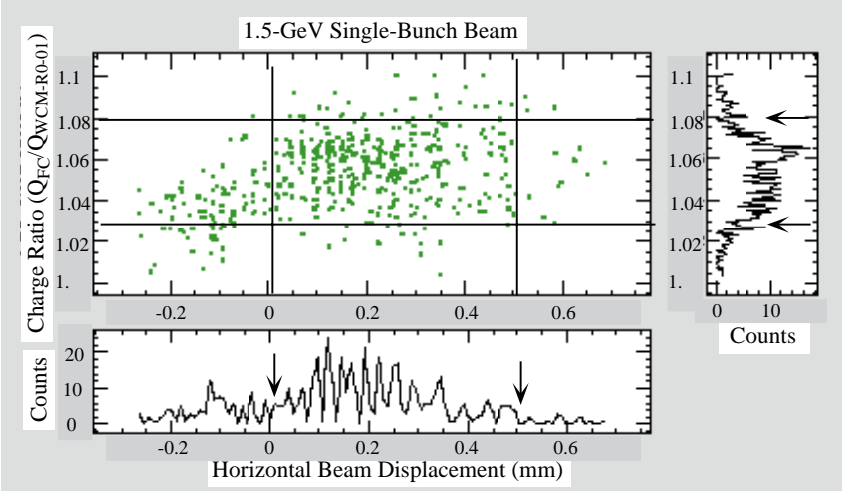

Figure 3: Scatter plots for the charge ratio $\left(\mathrm{Q}_{\mathrm{FC}} / \mathrm{Q}_{\mathrm{WCM}-\mathrm{R0} 0 \text {-01 }}\right)$ to the horizontal beam displacement measured for the1.5$\mathrm{GeV}$ single-bunch electron beam. The allows and lines indicate the data-reduction conditions.

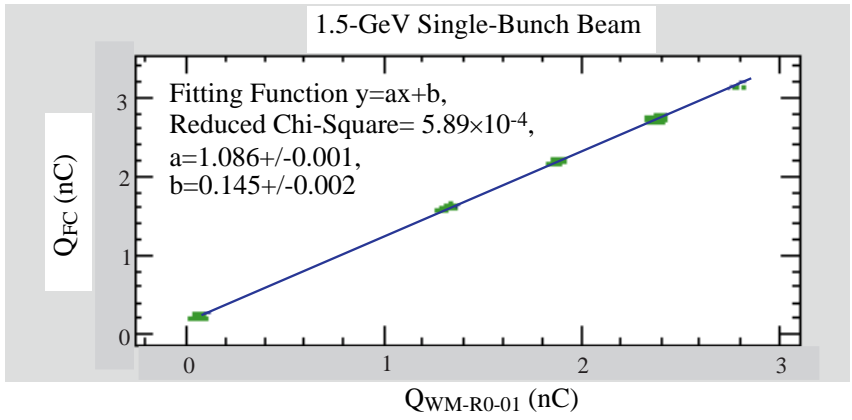

Figure 4: Linear relation of the beam charge measured by the WCM-R0-01 to the FC for the $1.5-\mathrm{GeV}$ single-bunch electron beam.

Table 1: Results for the beam experiment. " $a$ " and " $\delta a$ " show the recalibration coefficient and its error, respectively.

\begin{tabular}{|l|c|c|}
\hline Beam Mode & $a$ & $\delta a$ (stat.,syst. $)$ \\
\hline 1.5-GeV s-b beam & 1.086 & $\pm 1 \times 10^{-3} \pm 2 \times 10^{-2}$ \\
\hline 1.5-GeV s-p beam & 1.031 & $\pm 2 \times 10^{-3} \pm 2 \times 10^{-2}$ \\
\hline 325-MeV s-b beam & 1.126 & $\pm 1 \times 10^{-3} \pm 2 \times 10^{-2}$ \\
\hline 325-MeV s-p beam & 1.061 & $\pm 3 \times 10^{-3} \pm 2 \times 10^{-2}$ \\
\hline Combined s-b beam & 1.11 & $\pm 7 \times 10^{-4} \pm 2 \times 10^{-2}$ \\
\hline Combined s-p beam & 1.04 & $\pm 2 \times 10^{-3} \pm 2 \times 10^{-2}$ \\
\hline
\end{tabular}

